\title{
$X(3872)$ as a four-quark state in a Dyson-Schwinger/Bethe-Salpeter approach
}

\author{
Paul C. Wallbott, ${ }^{1, *}$ Gernot Eichmann, ${ }^{2, \dagger}$ and Christian S. Fischer ${ }^{1, \$}$ \\ ${ }^{1}$ Institut für Theoretische Physik, Justus-Liebig Universität Gießen, 35392 Gießen, Germany \\ ${ }^{2}$ CFTP, Instituto Superior Técnico, Universidade de Lisboa, 1049-001 Lisboa, Portugal
}

(Received 17 May 2019; published 30 July 2019)

\begin{abstract}
We generalize the framework of Dyson-Schwinger and Bethe-Salpeter equations for four-quark states to accommodate the case of unequal quark masses. As a first application, we consider the quantum numbers $I\left(J^{P C}\right)=0\left(1^{++}\right)$of the $X(3872)$ and study the four-quark states with quark contents $c q \bar{q} \bar{c}$ and $\operatorname{css} \bar{c}$. Their Bethe-Salpeter amplitudes are represented by a basis of heavy-light meson-meson, hadrocharmonium and diquark-antidiquark operators, which allows for a dynamical distinction between different internal configurations. In both cases we find the heavy-light meson-meson component to be dominant. For the putative $X(3872)$ we obtain a mass of $3916(74) \mathrm{MeV}$; the corresponding $c s \bar{s} \bar{c}$ state is predicted at 4068(61) MeV.
\end{abstract}

DOI: 10.1103/PhysRevD.100.014033

\section{INTRODUCTION}

With the spectacular success of Belle, BABAR, BES III and the LHC experiments and their discovery of an ever increasing and largely unexplained number of potentially exotic states, hadron spectroscopy in the heavy-quark region has become a fascinating topic in recent years; see e.g. [1-6] for recent reviews. This started out in 2003, when the Belle Collaboration found an unexpected and surprisingly narrow state in the $\mathrm{J} / \psi \pi^{+} \pi^{-}$invariant mass spectrum called the $X(3872)$ [7]. Since then this state has also been seen by other experiments [8-11] and its original quantum number assignment of $J^{P C}=1^{++}$was later confirmed by the LHCb Collaboration [12]. It turned out that the $X(3872)$ is hard to reconcile with a conventional charmonium meson. Its position very close to the $D \bar{D}$ threshold is remarkable as well as the fact that it decays into $\mathrm{J} / \psi \pi^{+} \pi^{-}$and $\mathrm{J} / \psi \pi^{+} \pi^{-} \pi^{0}$ with similar rates. The study of this object is a challenge for both theoretical and experimental groups; a detailed line shape analysis will be possible in the future PANDA experiment at FAIR [13].

From a theoretical perspective there are many open questions concerning the internal structure of exotic states. In this work we focus on four-quark states, i.e., states consisting of two quarks and two antiquarks in an overall color-singlet configuration. Moreover we specialize on

\footnotetext{
*paul.wallbott@physik.uni-giessen.de gernot.eichmann@tecnico.ulisboa.pt *christian.fischer@physik.uni-giessen.de
}

Published by the American Physical Society under the terms of the Creative Commons Attribution 4.0 International license. Further distribution of this work must maintain attribution to the author(s) and the published article's title, journal citation, and DOI. Funded by SCOAP. configurations with a heavy $c \bar{c}$ pair and a strange or light $q \bar{q}$ pair of valence quarks. These four quarks may (or may not) arrange themselves in subclusters. When the mass of the four-quark state is close to an open charm threshold it is plausible to assume an internal structure of a meson molecule [5]. The four quarks then arrange themselves into pairs of $D^{(*)} / \bar{D}^{(*)}$ mesons interacting with each other by short- and/or long-range forces. Naturally, such a picture makes most sense if the width of the constituents is smaller than the width of the four-quark state. The abovementioned $X(3872)$ has been considered as a prime candidate for such a state. Another possibility is the internal structure of a hadroquarkonium [14], where the heavy quark and antiquark group together in a core surrounded by the lighter $q \bar{q}$ pair. This picture is motivated by the observation that several potentially exotic hadrons were only discovered in final states of a specific charmonium state with light hadrons. It then seems natural to assume that the decay products are already preformed inside the four-quark state. Finally, four-quark states have been described as bound objects clustered in diquark-antidiquark $(d q-\overline{d q})$ components ${ }^{1}$ [1]. In principle, the three different possibilities of internal clustering are not mutually exclusive. It may be that some of the experimentally observed states fall into one of the three categories, whereas others fall into another. It is therefore vital to develop theoretical approaches that can deal with all of the different possibilities.

Most effective field theory and model approaches to meson molecules, hadroquarkonia and $d q-\overline{d q}$ tetraquarks already

\footnotetext{
${ }^{1}$ Sometimes in the literature the term "tetraquark" is reserved for these configurations only; sometimes it is used for any fourquark state regardless of its internal structure. In this work we adopt the latter terminology.
} 
assume a certain internal structure from the start. This is different for lattice calculations, which work directly with the underlying QCD Lagrangian; see e.g. [15-24] and references therein. Lattice calculations of four-quark states are extremely expensive and thus it seems fair to say that at least in the charmquark energy range they are still performed at an exploratory level at small volumes, coarse lattices and using light quarks with masses larger than the physical point. Nevertheless, a number of interesting observations have been made $[18,19]$ : (i) in the isospin $I=0$ channel corresponding to the experimentally observed $X(3872)$ a state has been found, whereas a corresponding state in the $I=1$ channel is absent; (ii) diquark interpolating operators have been found to play a negligible role, whereas the presence of $c \bar{c}$ and $D \bar{D}^{*}$ operators was crucial.

In this work we present another approach that is able to deal with different internal configurations in one framework. The authors of Ref. [25] employed the functional approach of Dyson-Schwinger equations (DSEs) and a four-body Bethe-Salpeter equation (BSE) to describe the lowest scalar meson octet and successfully reproduced the mass hierarchy of the $f_{0}(500)$, the $\kappa$ and the $f_{0} / a_{0}(980)$. To this end, a special role of internal meson-meson configurations in the pseudoscalar meson channels has been identified: the strong binding in these channels due to dynamical chiral symmetry breaking induces a drastic reduction of the mass of the fourbody states from the natural scale of $1300-1500 \mathrm{MeV}$ (four valence quarks) down to a mass of roughly $400-500 \mathrm{MeV}$ for the $f_{0}(500)$. The dominant role of meson-meson configurations also entails that $d q-\overline{d q}$ clusters were found to have a negligible impact.

In the present work we generalize the framework of Ref. [25] in two respects. First, we consider quarks with unequal masses, thus accommodating the cases of $c q \bar{q} \bar{c}$ and $\operatorname{cs} \bar{s} \bar{c}$ quark flavors. Secondly, we consider the quantum numbers $1^{++}$of the $X(3872)$ channel. We study this state in a spin-flavor basis which includes all three internal configurations: pairs of heavy-light mesons, hadrocharmonium and $d q-\overline{d q}$, letting the dynamics decide which of these structures is favored.

The paper is organized as follows. Section II deals with the technical setup of the framework. We briefly summarize the derivation of the four-body equation. We also discuss the truncation of the two-body interactions and the construction of the basis for the Bethe-Salpeter amplitude. Some technical details are relegated to the Appendix. In Sec. III we then discuss our results before we conclude in Sec. IV.

\section{FOUR-QUARK STATES IN THE DSE/BSE APPROACH}

\section{A. Four-body Bethe-Salpeter equation}

Four-quark states in QCD must appear as poles in the $q q \bar{q} \bar{q}$ scattering matrix $T$, which is an eight-point correlation function and satisfies the scattering equation

$$
T=K+K G_{0} T .
$$

Here, $K$ is the four-quark interaction kernel and $G_{0}$ the product of four dressed (anti)quark propagators. In this compact notation, each multiplication represents an integration over all loop momenta. The poles in the scattering matrix appear for real or complex values of the total momentum transfer $P^{2}$. At a given pole, the residue of the scattering equation is the homogeneous BSE for the fourquark Bethe-Salpeter (BS) amplitude $\Gamma$ shown in Fig. 1:

$$
\Gamma=K G_{0} \Gamma \text {. }
$$

In general, Eq. (2) is an eigenvalue equation for $K G_{0}$ whose eigenvalues $\lambda_{i}\left(P^{2}\right)$ depend on $P^{2} \in \mathbb{C}$. If an eigenvalue satisfies $\lambda_{i}\left(P_{i}^{2}\right)=1$, this corresponds to a pole in the scattering matrix with $P_{i}^{2}=-M_{i}^{2}$. If the mass $M_{i}$ is real and lies below a given meson-meson threshold, it describes a bound state; for a resonance the condition can only be satisfied in the complex plane of a higher Riemann sheet. Either way, in principle the homogeneous BSE is suitable to detect both bound states and resonances, although the calculation of $\lambda_{i}\left(P^{2}\right)$ above the lowest threshold requires contour deformations and direct access to the second sheet depends on knowledge of the full scattering amplitude [26].

The exact kernel $K$ in Eq. (1) is the sum of two-, threeand four-body irreducible interactions. In the following we neglect three- and four-body forces, so that the resulting kernel is the sum of two-body interactions:

$$
K G_{0}=\sum_{a a^{\prime}} K_{a a^{\prime}}
$$

where $a, a^{\prime}$ denote $q q, \bar{q} \bar{q}$ or $q \bar{q}$ pairs and $a a^{\prime}$ is one of the three combinations (12)(34), (13)(24) or (14)(23). $K_{a a^{\prime}}$ then describes the component of the four-body kernel where all interactions are switched off except those within the pairs $a$ and $a^{\prime}$. The resulting equation is shown in Fig. 1 and can be rewritten as a Faddeev-Yakubovski equation [27]. The particular form of the kernel

$$
K_{a a^{\prime}}=K_{a}+K_{a^{\prime}}-K_{a} K_{a^{\prime}}
$$

avoids overcounting and ensures the separability of the four-body correlation function obtained from one channel

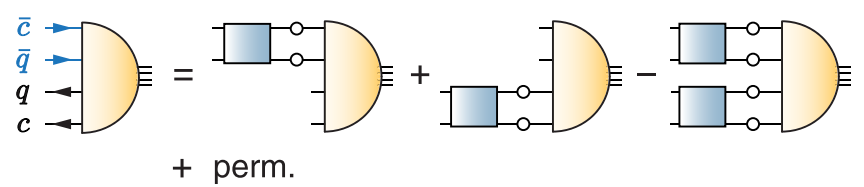

FIG. 1. Four-quark BSE for a $c q \bar{q} \bar{c}$ system in the (12)(34) configuration; the remaining (13)(24) and (14)(23) permutations are not shown. The half-circles and boxes represent the tetraquark amplitude and Bethe-Salpeter kernel, respectively. 
$a a^{\prime}$ only and thus the absence of residual color forces between widely separated clusters [28-30].

We employ a rainbow-ladder kernel for the $q \bar{q}$ and $q q$ interaction, which amounts to an iterated dressed gluon exchange and has been reviewed recently [31] together with more advanced schemes; see also [32,33] for details. The construction satisfies chiral constraints such as the Gell-Mann-Oakes-Renner relation and ensures the (pseudo-)Goldstone-boson nature of the pion. The construction has been extensively applied to meson and baryon phenomenology. To this end one defines an effective interaction $\alpha\left(k^{2}\right)$ which incorporates dressing effects of the gluon propagator and quark-gluon vertex [34]; once specified, all further elements of the calculation follow. We solve the quark DSE for a range of quark masses and their resulting dressed propagators enter in the kernel $K G_{0}$. The input current-quark masses for up/down, strange and charm are listed in Table I.

\section{B. Four-quark amplitude}

The BS amplitude of a four-quark state with quantum numbers $J^{P C}=1^{++}$is the direct product of Dirac, color and flavor parts:

$$
\Gamma^{\mu}(p, q, k, P)=\Gamma_{\mathrm{D}}^{\mu}(p, q, k, P) \otimes \Gamma_{\mathrm{C}} \otimes \Gamma_{\mathrm{F}} .
$$

From $3 \otimes \mathbf{3} \otimes \overline{\mathbf{3}} \otimes \overline{\mathbf{3}}=(\overline{\mathbf{3}} \oplus \mathbf{6}) \otimes(\mathbf{3} \oplus \overline{\mathbf{6}})=\mathbf{1} \oplus \mathbf{1} \oplus \ldots$, the color part of the amplitude consists of two independent color singlet tensors, which can be picked from the $d q-\overline{d q}$ $(\overline{\mathbf{3}} \otimes \mathbf{3}, \mathbf{6} \otimes \overline{\mathbf{6}})$ or either of the meson-meson configurations $(1 \otimes \mathbf{1}, \mathbf{8} \otimes \mathbf{8})$, for example:

$$
\left(\mathcal{C}_{11}\right)_{A B C D}=\frac{1}{3} \delta_{A C} \delta_{B D} \quad\left(\mathcal{C}_{11}^{\prime}\right)_{A B C D}=\frac{1}{3} \delta_{A D} \delta_{B C} .
$$

The two tensors in the $d q-\overline{d q}$ decomposition are linear combinations of these,

TABLE I. Rainbow-ladder results for $q \bar{q}, c \bar{q}, c \bar{s}$ and $c \bar{c}$ meson and diquark masses (in MeV). $m_{\bar{q}}$ is the input current-quark mass at a renormalization point $\mu=19 \mathrm{GeV}$ in a momentum subtraction scheme. The column $m_{P S}$ contains the masses of $\pi, D, D_{s}$ and $\eta_{c}$; the column $m_{V}$ those of $\rho / \omega, D^{*}, D_{s}^{*}$ and $J / \psi$; and the columns $m_{S}$ and $m_{A}$ list the corresponding diquark masses. The errors quoted are obtained by varying the parameter $\eta=1.8 \pm 0.2$.

\begin{tabular}{cccccc}
\hline \hline & $m_{\bar{q}}$ & $m_{P S}$ & $m_{V}$ & $m_{S}$ & $m_{A}$ \\
\hline$q \bar{q}$ & 3.7 & $138(3)$ & $732(1)$ & $802(77)$ & $999(60)$ \\
$c \bar{q}$ & 3.7 & $1802(2)$ & $2068(16)$ & $2532(90)$ & $2572(8)$ \\
$c \bar{s}$ & 91 & $1911(3)$ & $2169(14)$ & $2627(82)$ & $2666(7)$ \\
$c \bar{c}$ & 795 & $2792(6)$ & $2980(6)$ & $3382(15)$ & $3423(8)$ \\
\hline \hline
\end{tabular}

$\mathcal{C}_{\overline{3} 3}=-\frac{\sqrt{3}}{2}\left(\mathcal{C}_{11}-\mathcal{C}_{11}^{\prime}\right), \quad \mathcal{C}_{6 \overline{6}}=\sqrt{\frac{3}{8}}\left(\mathcal{C}_{11}+\mathcal{C}_{11}^{\prime}\right)$,

as well as the remaining octet-octet tensors:

$$
\mathcal{C}_{88}=\frac{\mathcal{C}_{11}-3 \mathcal{C}_{11}^{\prime}}{2 \sqrt{2}}, \quad \mathcal{C}_{88}^{\prime}=\frac{\mathcal{C}_{11}^{\prime}-3 \mathcal{C}_{11}}{2 \sqrt{2}}
$$

The tensors $\left\{\mathcal{C}_{11}, \mathcal{C}_{88}\right\},\left\{\mathcal{C}_{11}^{\prime}, \mathcal{C}_{88}^{\prime}\right\}$ and $\left\{\mathcal{C}_{\overline{3} 3}, \mathcal{C}_{6 \overline{6}}\right\}$ are mutually orthogonal.

Concerning flavor, a four-quark state made of $c q \bar{q} \bar{c}$ allows for several flavor wave functions. For further use we collect the combinations

$$
\begin{aligned}
\mathcal{F}_{0} & =c u \bar{u} \bar{c}+c d \bar{d} \bar{c}, \\
\mathcal{F}_{1} & =[c u]\{\bar{u} \bar{c}\}+[c d]\{\bar{d} \bar{c}\}, \\
\mathcal{F}_{2} & =\{c u\}[\bar{u} \bar{c}]+\{c d\}[\bar{d} \bar{c}],
\end{aligned}
$$

where $\{\ldots\}$ and $[\ldots]$ denote symmetrization and antisymmetrization, respectively.

The Dirac part $\Gamma_{\mathrm{D}}^{\mu}$ depends on the total momentum $P$ and the relative momenta $p, q$ and $k$. They are related to the individual (outgoing) quark momenta $p_{i}$ via

$\begin{array}{ll}p_{1}=\frac{k+q-p}{2}+\sigma_{1} P, & p_{2}=\frac{k-q+p}{2}+\sigma_{2} P, \\ p_{3}=\frac{-k+q+p}{2}+\sigma_{3} P, & p_{4}=\frac{-k-q-p}{2}+\sigma_{4} P,\end{array}$

where $0 \leq \sigma_{i} \leq 1$ is a set of momentum partitioning parameters satisfying $\sum_{i=1}^{4} \sigma_{i}=1$. The most general decomposition of $\Gamma_{\mathrm{D}}^{\mu}$ with one vector index and four Dirac indices involves 768 linearly independent tensors $\tau_{i}^{\mu}$, which are collected in the Appendix:

$$
\Gamma_{\mathrm{D}}^{\mu}(p, q, k, P)=\sum_{i=1}^{768} f_{i}(\Omega) \tau_{i}^{\mu}(p, q, k, P) .
$$

The scalar dressing functions $f_{i}$ depend on the nine Lorentz invariants obtained by combining the four-vectors $p, q, k$ and $P: \Omega=\left\{p^{2}, q^{2}, k^{2}, p \cdot q, \ldots\right\}$, with $P^{2}=-M^{2}$ fixed. Herein lies the main challenge in solving the tetraquark BSE numerically: Eq. (2) produces a set of coupled integral equations for the dressing functions $f_{i}(\Omega)$ which depend on nine independent variables each. This complexity makes calculations tedious and numerically challenging.

In Ref. [25] these nine Lorentz invariants were mapped onto a set of permutation-group variables [35], which form a singlet $\mathcal{S}_{0}$ and a doublet $\mathcal{D}$, 


$$
\mathcal{S}_{0}=\frac{p^{2}+q^{2}+k^{2}}{4}, \quad \mathcal{D}=\frac{1}{4 \mathcal{S}_{0}}\left(\begin{array}{c}
\sqrt{3}\left(q^{2}-p^{2}\right) \\
p^{2}+q^{2}-2 k^{2}
\end{array}\right),
$$

as well as two triplets $\mathcal{T}_{0}$ and $\mathcal{T}_{1}$, under transformations of the permutation group $S_{4}$. This ordering scheme allows one to take into account or discard the dependence of the amplitude on groups of variables (the members of the multiplets) without destroying its symmetries and turned out to be crucial with respect to numerical feasibility.

In Ref. [25] it was found that light scalar tetraquarks mainly depend on the three variables encoded in $\mathcal{S}_{0}$ and $\mathcal{D}$. Retaining $\mathcal{S}_{0}$ only, their masses are in the ballpark of what one would naively expect for a state made of four quarks ( $\sim 1500 \mathrm{MeV}$ for four light quarks). However, the fourquark BSE dynamically and self-consistently generates intermediate meson-meson and $d q-\overline{d q}$ poles in the dressing functions $f_{i}$ corresponding to the (12)(34), (13)(24) and (14)(23) topologies. These poles appear in the Mandelstam plane formed by the doublet variables $\mathcal{D}$ and produce decay thresholds. Due to the lightness of the pions and kaons as a consequence of spontaneous chiral symmetry breaking, they induce a sizable mass shift by almost $1 \mathrm{GeV}$, such that the bound state around $1500 \mathrm{MeV}$ turns into a resonance at a mass scale of about $400-500 \mathrm{MeV}$. Therefore, the implicit resonance mechanism and the possibility of a tetraquark to decay into light pseudoscalar mesons reduce its mass and lead to a mass pattern which is similar to the mass ordering for the $\sigma, \kappa$ and $a_{0} / f_{0}$ observed in experiment.

While the permutation-group method greatly simplifies the problem, the dynamical creation of intermediate twobody poles still causes numerical complications. The eigenvalues of Eq. (2) are calculated below all thresholds where the poles do not yet enter in the integration domain, but the rapid variation of the dressing functions in their vicinity makes numerical calculations cumbersome. Moreover, tracking the resonance locations in the complex plane would require us to go above those thresholds using contour deformations [26,36,37], where one additionally has to circumvent dynamically generated moving poles that only emerge in the solution of the equation itself. For these reasons we aim for another simplification in what follows and absorb the intermediate particle poles into the tensor basis of the amplitude.

\section{Physically motivated tensor basis}

To describe an axialvector tetraquark with quark content $c q \bar{q} \bar{c}$ and quantum numbers $I\left(J^{P C}\right)=0\left(1^{++}\right)$in terms of meson-meson and $d q-\overline{d q}$ compositions, we consider the following combinations based on the two-particle decay modes of the $X(3872)$ [38]:

(i) $D_{a c}^{0} \bar{D}_{b d}^{* 0}+D_{a c}^{* 0} \bar{D}_{b d}^{0}+D_{a c}^{+} D_{b d}^{*-}+D_{a c}^{*+} D_{b d}^{-}$,

(ii) $J / \Psi_{a d} \omega_{b c}$,

(iii) $S_{a b} A_{c d}+A_{a b} S_{c d}$.
The first is the composition in terms of a $D \bar{D}^{*}$ molecule, which is motivated by the proximity of the $X(3872)$ to the $D \bar{D}^{*}$ threshold. By contrast, the option $D \bar{D}$ can produce $J=1$ only with nonvanishing orbital angular momentum. Second, a hadrocharmonium configuration containing a $J / \Psi$ with $0\left(1^{--}\right)$would require a light pseudoscalar state with exotic quantum numbers $0\left(0^{--}\right)$. Since such a state is not observed, this leaves the vector state $\omega$ with $0\left(1^{--}\right)$as a possible partner. Finally, viewed as a $d q-\overline{d q}$ state, the "good" diquarks are the scalar diquarks $(S)$ with $J^{P}=0^{+}$ and the axialvector diquarks $(A)$ with $J^{P}=1^{+}$, which are both in a color-antitriplet configuration so that the colorsinglet tensor is $\mathcal{C}_{\overline{3} 3}$.

Based on this, we approximate the Dirac-color-flavor structure of the BS amplitude as

$$
\Gamma^{\mu}(p, q, k, P) \approx \sum_{i=1}^{3} f_{i}\left(\mathcal{S}_{0}\right) T_{i}^{\mu},
$$

where each $T_{i}^{\mu}$ corresponds to one of the compositions above:

$$
\begin{aligned}
T_{1}^{\mu} & =R_{1}^{\mu} \mathcal{C}_{11} \mathcal{F}_{0}, \\
T_{2}^{\mu} & =R_{2}^{\mu} \mathcal{C}_{11}^{\prime} \mathcal{F}_{0}, \\
T_{3}^{\mu} & =\left(R_{3}^{\mu}-R_{4}^{\mu}\right) \mathcal{C}_{\overline{3} 3} \mathcal{F}_{0} .
\end{aligned}
$$

Here, $\mathcal{C}_{11}, \mathcal{C}_{11}^{\prime}$ and $\mathcal{C}_{\overline{3} 3}$ are the color tensors defined in Eqs. (6)-(7) and $\mathcal{F}_{0}$ is the $I=0$ flavor tensor from Eq. (9). With the meson flavor wave functions given by $\left\{D^{0}\right.$, $\left.\bar{D}^{0}, D^{+}, D^{-}, J / \psi, \omega\right\} \sim\{c \bar{u}, u \bar{c}, c \bar{d}, d \bar{c}, c \bar{c}, u \bar{u}+d \bar{d}\}, \mathcal{F}_{0}$ emerges automatically in the construction of the mesonmeson components. The Dirac parts $R_{i}^{\mu}$ are

$$
\begin{aligned}
R_{1}^{\mu} & =\left[P\left(m_{D}, m_{D}^{*}\right) \gamma_{5} \otimes \gamma_{\perp}^{\mu}-P\left(m_{D}^{*}, m_{D}\right) \gamma_{\perp}^{\mu} \otimes \gamma_{5}\right]_{a c, b d}, \\
R_{2}^{\mu} & =\left[P\left(m_{J / \Psi}, m_{\omega}\right) \gamma^{\alpha} \otimes \gamma^{\beta} \hat{P}^{\nu} \epsilon^{\alpha \beta \nu \mu}\right]_{a d, b c}, \\
R_{3}^{\mu} & =\left[P\left(m_{S}, m_{A}\right) \gamma_{5} C \otimes C^{T} \gamma_{\perp}^{\mu}\right]_{a b, c d}, \\
R_{4}^{\mu} & =\left[P\left(m_{A}, m_{S}\right) \gamma_{\perp}^{\mu} C \otimes C^{T} \gamma_{5}\right]_{a b, c d},
\end{aligned}
$$

where $\gamma_{\perp}^{\mu}=\gamma_{\mu}-\hat{P} \hat{P}^{\mu}$ is the transverse $\gamma$-matrix, $\hat{P}$ the normalized total momentum, $C=\gamma_{4} \gamma_{2}$ the charge conjugation matrix and $T$ denotes a matrix transpose. The multiindices $(a, b, c, d)$ stand for Dirac indices as well as momentum labels.

In Eq. (15) we have absorbed the two-body poles, which would emerge dynamically in the solution of the four-body equation, directly into the tensor structure by defining

$$
P\left(m_{1}, m_{2}\right)_{a b, c d}=\frac{1}{\left(p_{a}+p_{b}\right)^{2}+m_{1}^{2}} \frac{1}{\left(p_{c}+p_{d}\right)^{2}+m_{2}^{2}} .
$$

Given that this form captures all relevant momentum dependencies beyond the symmetric variable $\mathcal{S}_{0}$, which 
turns out to be a good approximation for the light scalar tetraquarks [25], the remaining dressing functions $f_{i}$ depend on $\mathcal{S}_{0}$ only. The pole masses $m_{D}, m_{D^{*}}, m_{J / \psi}$, $m_{\omega}, m_{S}$ and $m_{A}$ are calculated from the corresponding twobody BSEs in rainbow-ladder truncation $[31,39,40]$ and collected in Table I. The explicit form of the two-body interaction used in the BSE for the heavy-light mesons but also in the four-body BSE is given in Eq. (3.96) of the review Ref. [31]. We use the typical value $\Lambda=0.72 \mathrm{GeV}$ for the scale parameter, matched to reproduce the experimental value of the pion decay constant $f_{\pi}$, and $\eta=1.8 \pm 0.2$. We work in the isospin symmetric limit where $m_{D^{+}}=m_{D^{-}}=m_{D^{0}}$. The charm quark mass is determined by the condition that the sum $m_{D}+m_{D^{*}}$ equal the sum of the experimental masses [38]. The strange quark mass is determined analogously for $m_{D_{s}}+m_{D_{s}^{*}}$.

In principle one could systematically proceed and construct a complete basis for the tetraquark amplitude with entangled Dirac, color and flavor tensors from all possible meson and diquark channels, and finally also restore the full momentum dependence of the dressing functions $f_{i}(\Omega)$. Our assumption here is that the amplitude is dominated by the three "physical" channels $D \bar{D}^{*}, J / \psi \omega$ and $d q-\overline{d q}$ and that all momentum dependencies of the amplitude except those in $\mathcal{S}_{0}$ can be absorbed in the $T_{i}^{\mu}$. This allows us to set $\mathcal{D}=\mathcal{T}_{0}=\mathcal{T}_{1}=0$ on the external momentum grid on the lhs of the tetraquark BSE (2), which in the rest frame of the total momentum $P$ entails

$$
\left\{k^{\mu}, p^{\mu}, q^{\mu}\right\}=\frac{2 \mathcal{S}_{0}}{\sqrt{3}}\left\{e_{1}^{\mu}, e_{2}^{\mu}, e_{3}^{\mu}\right\}, \quad P^{\mu}=i M e_{4}^{\mu},
$$

where $e_{i}^{\mu}$ are the Euclidean unit vectors. The rhs of (2) still samples the full domain of $\Omega$ under the integral.

Concerning the $d q-\overline{d q}$ flavor structure in Eq. (14), one can alternatively consider the tensor

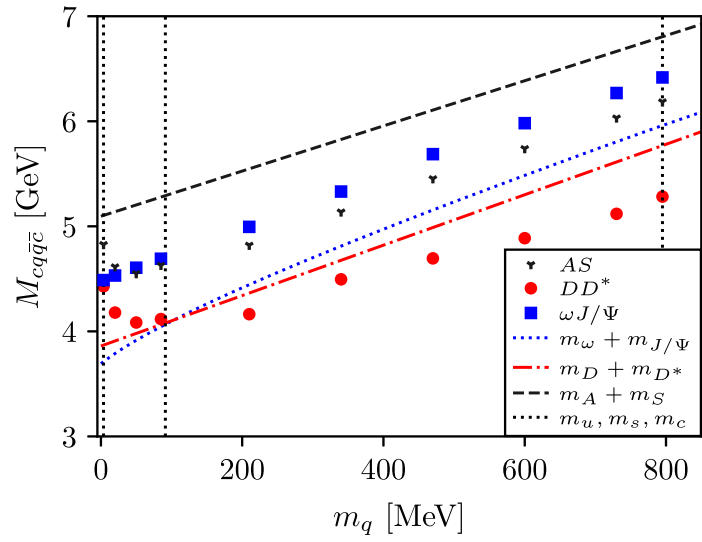

$$
\tilde{T}_{3}^{\mu}=\left(R_{4}^{\mu} \mathcal{F}_{2}-R_{3}^{\mu} \mathcal{F}_{1}\right) \mathcal{C}_{\overline{3} 3},
$$

where the Pauli antisymmetry of the diquarks [which would hold for exact $S U(4)$ flavor symmetry] is explicit. Combined with the antisymmetric color tensor $\sim \varepsilon_{A B C}$, the antisymmetric tensor $\gamma_{5} \mathrm{C}$ for a scalar diquark is matched with an antisymmetric flavor structure and the symmetric tensor $\gamma^{\mu} C$ for the axialvector diquarks with a symmetric flavor wave function. In contrast to Eq. (14), however, after plugging Eq. (17) into the BSE and taking flavor traces the diquarks decouple from the meson-meson configurations in this case, so that both can be solved independently and yield two different states. This setup requires no separate discussion since the decoupled $d q-\overline{d q}$ state is identical for $T_{3}$ and $\tilde{T}_{3}$ and the equations for the meson-meson configuration are the same when dropping the diquarks in the construction of the amplitude in Eq. (13), which we will do below for comparison.

\section{RESULTS}

Before discussing the results, we briefly summarize our setup. We solve the four-body equation depicted in Fig. 1 with the rainbow-ladder kernel including all permutations, where the dressed light and charm-quark propagators are obtained from their DSEs. We approximate the structure of the tetraquark amplitude by its three dominant components in Eq. (14), which has the advantage of reducing the complexity of the four-body equation while the system still dynamically decides which of the three configurationsheavy-light meson, hadrocharmonium or $d q-\overline{d q}$-is most important.

In Fig. 2 we track the tetraquark mass as a function of the light current-quark mass $m_{q}$, which we vary from the charm-quark mass $(c c \bar{c} \bar{c})$ down to the up/down quark mass $(c q \bar{q} \bar{c})$. At each quark mass and for each setup we calculate the eigenvalues $\lambda_{i}\left(P^{2}\right)$ of the kernel of the tetraquark BSE

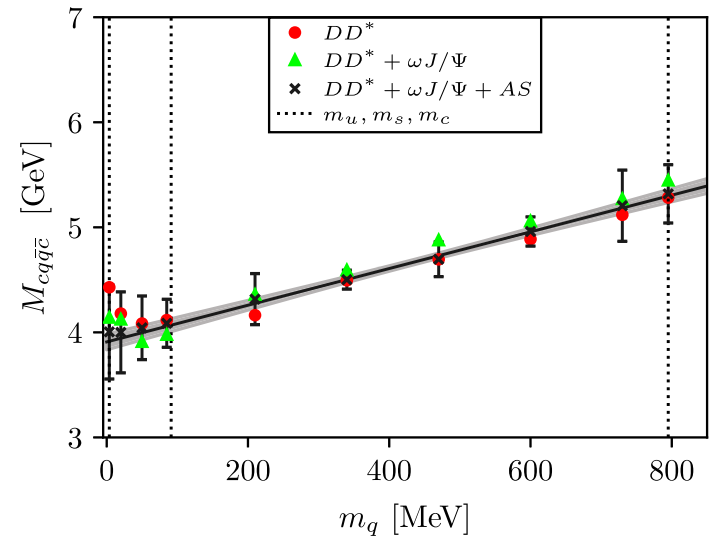

FIG. 2. Mass of the $I\left(J^{P C}\right)=0\left(1^{++}\right)$four-quark state as a function of the current-quark mass. Left: Solutions for the individual $D \bar{D}^{*}$, $J / \psi \omega$ and diquark-antidiquark components together with their respective thresholds. Right: Results obtained from including one $\left(D \bar{D}^{*}\right)$, two and all three channels. The error bars combine the extrapolation error with the error obtained by varying the momentum partitioning parameter $\zeta$. The black line is a fit to the data points together with an error band. 
(2) below the lowest-lying two-particle decay threshold, i.e., for $P^{2}>-M_{\mathrm{thr}}^{2}$ along the real axis. We track the largest eigenvalue as a function of $P^{2}$ and read off the mass $M$ of the ground state from the condition $\lambda_{0}\left(P^{2}=-M^{2}\right)=1$. In cases where this condition is not satisfied below the threshold, we extrapolate the eigenvalue to obtain an estimate for the real part of the resonance mass, although one should be cautious when interpreting results obtained from extrapolating over thresholds [41]. As explained earlier, we cannot yet access the whole complex $P^{2}$ plane due to restrictions imposed by the intermediate meson and diquark poles as well as the poles in the complex plane of the quark propagator, which would require contour deformations.

In the left panel of Fig. 2 we plot the results obtained for each of the three configurations in Eq. (13) separately: $D \bar{D}^{*}, J / \psi \omega$ and $d q-\overline{d q}$, together with their respective thresholds. The thresholds for $D \bar{D}^{*}$ and $J / \psi \omega$ are relatively close to each other, whereas the sum of the scalar and axialvector diquark masses obtained from their BSEs is larger by almost $1 \mathrm{GeV}$. This already provides a first indication that the diquark contributions may be subleading compared to the meson-meson components simply due to their larger masses, similarly as in the case of the light scalar mesons [25].

This is indeed what we observe in the left panel of Fig. 2: Solved for each individual tensor $T_{1}, T_{2}$ or $T_{3}$ alone, it turns out that the $D \bar{D}^{*}$ component has the lowest mass, followed by the $d q-\overline{d q}$ and the $J / \psi \omega$ components. The $d q-\overline{d q}$ mass is always below its threshold, whereas the $D \bar{D}^{*}$ mass lies below threshold only above the strange-quark mass and the $J / \psi \omega$ mass comes out above its threshold. In the right panel of Fig. 2 we show again the result for the $D \bar{D}^{*}$ component $T_{1}$ together with the results including both $T_{1}$ and $T_{2}$ and the full result with all three tensors. Indeed one can see that the addition of the $d q-\overline{d q}$ tensors and the $J / \psi \omega$ component has little effect and the mass of the state is essentially determined by the $D \bar{D}^{*}$ component alone.

To provide an estimate of the error of the calculation we vary the momentum partitioning parameters in Eq. (10). Had we included all kinematic variables, i.e. all permutation group multiplets discussed above, the results would be independent of the momentum partitioning; however, with a smaller subset this is no longer the case. In practice we choose $\sigma_{1}=\sigma_{4}=\frac{1}{2}-\zeta$ and $\sigma_{2}=\sigma_{3}=\zeta$, which leaves one parameter $\zeta$ that quantifies the fraction of the momentum $P$ assigned to the $c \bar{c}$ and $q \bar{q}$ pairs. We then optimize that value to minimize the distance to the thresholds and vary $\zeta$ in the vicinity of its optimal value. The errors obtained in this case are shown in the first row of Table II. One clearly sees that the error increases with decreasing light quark mass $m_{q}$. The second row shows the error obtained by optimizing the momentum partitioning without restricting the $\sigma_{i}$, which has a similar but even larger effect.
TABLE II. Error estimates from the momentum partitioning for one parameter $\zeta$ (row 1) and without restricting the $\sigma_{i}$ (row 2).

$\begin{array}{lllllllllll}m_{q}(\mathrm{MeV}) & 3.7 & 20 & 50 & 85 & 210 & 340 & 470 & 600 & 730 & 795\end{array}$

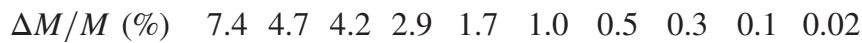

\begin{tabular}{lllllllllll}
$\Delta M / M(\%)$ & 10.1 & 9.2 & 7.4 & 5.5 & 5.8 & 2.0 & 3.5 & 2.8 & 6.6 & 5.3 \\
\hline \hline
\end{tabular}

The rise of the tetraquark masses in Fig. 2 towards smaller quark masses (even for points below thresholds) is thus an artifact of the reduced kinematics, which induces an error of at least $10 \%$ at the physical $u / d$ mass.

Within errors, all three results of Fig. 2 are the same and dominated by the heavy-light meson $D \bar{D}^{*}$ component, whereas the $d q-\overline{d q}$ and $J / \psi \omega$ components are almost negligible. The black solid line in the figure is obtained by fitting the linear expression

$$
M=c_{0}+c_{1} m_{q}
$$

to the six data points at the larger current-quark masses where the tetraquark is definitely a bound state. The grey band indicates the combined errors from the momentum partitioning and-for the four lowest quark masses-the extrapolation error. At the physical $u / d$ quark mass, the fit yields

$$
M_{1^{++}}^{c q \bar{q} \bar{c}}=3916(74) \mathrm{MeV}
$$

for the mass of the axialvector tetraquark in good agreement with the mass of the $X(3872)$. In addition we find

$$
M_{1^{++}}^{c s \bar{c} \bar{c}}=4068(61) \mathrm{MeV}
$$

for the mass of a putative four-quark state with charm and strange quarks. Note that from Fig. 2 one cannot read off the mass of an all-charm tetraquark since the implemented symmetries among the various constituents are no longer appropriate when all four quarks are equal.

\section{CONCLUSIONS}

When solving the four-body equation for a system of heavy-light quark flavors in the $I\left(J^{P C}\right)=0\left(1^{++}\right)$channel, we find a similar behavior as for the light scalar meson octet discussed in Refs. [25,30]: The ground state is dominated by a strong meson-meson component. For the light scalar mesons this component has pseudoscalar quantum numbers and is strongly affected by the effects of dynamical chiral symmetry breaking. For the case considered here, it is a combination of heavy-light pseudoscalar and vector components, namely the $D \bar{D}^{*}$ combination. In both cases, diquark-antidiquark components in the wave function are negligible. For the case of heavy-light four-quark states one can in addition identify a hadrocharmonium contribution to 
the wave function which, however, is also subdominant as compared to the dominating heavy-light $D \bar{D}^{*}$ component.

The precision of our calculation is not good enough to decide whether the resulting axialvector four-body state is a bound meson molecule or not - to this end we would need to be able to determine its mass on the several-MeV level, which is not possible with the tools at hand. Nevertheless, within error bars, we find a state in the correct mass range to be identified with the $X(3872)$. In order to corroborate our findings we need to gain precision using appropriate techniques to deal with the analytic structure of the fourbody equation beyond extrapolations. Furthermore it would be very interesting to include a $c \bar{c}$ component in the wave function in order to address mixing with ordinary charmonia. These improvements are subject to future work.

\section{ACKNOWLEDGMENTS}

We are grateful to Christoph Hanhart, Soeren Lange and Marc Wagner for discussions. This work was supported by the Helmholtz International Center for FAIR within the LOEWE program of the State of Hesse, by the DFG Grant No. FI 970/11-1 and by the FCT Investigator Grant No. IF/ 00898/2015.

\section{APPENDIX: TENSOR BASIS}

The axialvector tetraquark amplitude in Eq. (11) depends on 768 Dirac tensor basis elements. To derive them, we first recapitulate the construction of the basis for a scalar tetraquark [25]. By orthogonalizing the momenta $p, q$, $k, P$ one obtains four orthonormal momenta $n_{i}^{\mu}(i=1 \ldots 4)$ that are mutually transverse. The set

$$
\left\{\mathbb{1}, \not h_{i}, \not h_{i} h_{j}, \not h_{i} h_{j} h_{k}, \not h_{i} h_{j} \not h_{k} h_{l}\right\}
$$

with $i<j<k<l$ consists of 16 elements; commutators are not necessary because $\not h_{i} h_{j}=-\not h_{j} h_{i}$. Taking all tensor products of Eq. (A1) with itself yields 256 linearly independent tensor structures. No further $\gamma$-matrices are necessary because they can be reconstructed from the unit vectors:

$$
\delta^{\mu \nu}=\sum_{i=1}^{4} n_{i}^{\mu} n_{i}^{\nu}, \quad \gamma^{\mu}=\sum_{i=1}^{4} n_{i}^{\mu} h_{i} .
$$

To further simplify (A1) we define the pseudoscalar

$$
\epsilon=\varepsilon^{\mu \nu \rho \sigma} n_{1}^{\mu} n_{2}^{\nu} n_{3}^{\rho} n_{4}^{\sigma}
$$

which can take values $\epsilon= \pm 1$. From the relation

$$
\frac{1}{24}\left[\gamma^{\mu}, \gamma^{\nu}, \gamma^{\alpha}, \gamma^{\beta}\right]=-\gamma_{5} \varepsilon^{\mu \nu \alpha \beta},
$$

where the four-commutator is the fully antisymmetrized product of four $\gamma$-matrices, one obtains

$$
\not h_{1} \not h_{2} \not h_{3} \not h_{4}=-\epsilon \gamma_{5} .
$$

In this way all elements in (A1) with three or four slashes can be reduced to those with two at most, so we can write it as $\left\{\mathbb{1}, \not h_{i}, \not h_{4}, \not h_{i} \not h_{4}\right\} \times \Omega_{\omega}$ with $\Omega_{1}=\mathbb{1} ; \Omega_{2}=\epsilon \gamma_{5} ;$ and $i=1,2$, 3. If $n_{4}^{\mu}=\hat{P}^{\mu}$ denotes the normalized total momentum and we express $\not h_{4}$ in terms of the positive/ negative-energy projectors $\Lambda_{ \pm}=\left(\mathbb{1} \pm \not h_{4}\right) / 2$, then (A1) becomes $\left\{\mathbb{1}, \not h_{i}\right\} \times \Lambda_{\lambda} \times \Omega_{\omega}$.

A complete, orthonormal, covariant, 256-dimensional positive-parity basis for the BS amplitude of a scalar tetraquark is then given by

$$
\tau_{n}(p, q, k, P)=\Gamma_{j} \Lambda_{\lambda} \Omega_{\omega} \gamma_{5} C \otimes C^{T} \gamma_{5} \Omega_{\omega^{\prime}} \Lambda_{\lambda^{\prime}} \Gamma_{k}
$$

with $\Gamma_{j} \in\left\{\mathbb{1}, \not h_{1}, \not h_{2}, \not h_{3}\right\}$. We inserted the combination $\gamma_{5} C \otimes C^{T} \gamma_{5}$ for the $d q-\overline{d q}$ topology (12)(34); all further structures such as $\gamma^{\mu} C \otimes C^{T} \gamma^{\mu}$ but also those in the mesonmeson topologies are linearly dependent.

Because the $\gamma$-matrices can be reduced to the unit vectors and their slashes according to Eq. (A2), an orthonormal basis for the axialvector tetraquark simply follows from attaching $\epsilon n_{i}^{\mu}$ with $i=1,2,3$ to (A6). $n_{4}^{\mu}$ cannot appear because the tetraquark must be transverse in the total momentum. This yields $3 \times 256=768$ linearly independent and covariant Lorentz-Dirac tensors.

In practice we are interested in partial-wave bases whose tensors are eigenstates of the total quark spin and orbital angular momentum in the tetraquark's rest frame. The construction is analogous to the nucleon's Faddeev amplitude [42]. The eigenvalues of the quark spin can take values $s=0,1,2$, which can combine with $l=0,1,2,3$ to produce total angular momentum $J=1$. The orbital angular momentum operator $L^{2}$ only acts on the unit vectors $n_{i}^{\mu}$ with $i=1,2,3$ but not on the total momentum $\sim n_{4}^{\mu}$, and it leaves Lorentz scalars as well as $\epsilon$ invariant. Thus we only need to consider the combinations $n_{i}^{\mu}\left\{\mathbb{1}, h_{j}\right\} \otimes\left\{\mathbb{1}, \not h_{k}\right\} . L^{2}$ also does not act on the Dirac structure, so the problem reduces to arranging

$$
n_{i}^{\mu}, \quad n_{i}^{\mu} n_{j}^{\alpha}, \quad n_{i}^{\mu} n_{k}^{\beta}, \quad n_{i}^{\mu} n_{j}^{\alpha} n_{k}^{\beta}
$$

into combinations that are eigenfunctions of $L^{2}$ with eigenvalues $l(l+1)$.

The resulting numbers of $s, p, d$ and $f$ waves are given in Table III. For example, the three unit vectors $n_{i}^{\mu}$ are $p$ waves because $L^{2} n_{i}^{\mu}=2 n_{i}^{\mu}$. The nine combinations $n_{i}^{\alpha} n_{j}^{\beta}$ produce one $s$ wave with $l=0$,

$$
T^{\alpha \beta}=\sum_{i=1}^{3} n_{i}^{\alpha} n_{i}^{\beta}=\delta^{\alpha \beta}-n_{4}^{\alpha} n_{4}^{\beta} ;
$$

three $p$ waves with $l=1$, 
TABLE III. Eigenfunctions of the orbital angular momentum operator obtained from combinations of the three unit vectors $n_{i}^{\mu}$, which correspond to the relative momenta $p, q$ and $k$.

\begin{tabular}{lllll}
\hline \hline & $s$ & $p$ & $d$ & $f$ \\
\hline$n_{i}^{\mu}$ & 0 & 3 & 0 & 0 \\
$n_{i}^{\mu} n_{j}^{\alpha}$ & 1 & 3 & 5 & 0 \\
$n_{i}^{\mu} n_{k}^{\beta}$ & 1 & 3 & 5 & 0 \\
$n_{i}^{\mu} n_{j}^{\alpha} n_{k}^{\beta}$ & 1 & 9 & 10 & 7 \\
\hline \hline
\end{tabular}

$$
n_{i}^{\alpha} n_{j}^{\beta}-n_{j}^{\alpha} n_{i}^{\beta}=\epsilon \varepsilon^{\alpha \beta \gamma \delta} n_{k}^{\gamma} n_{4}^{\delta},
$$

where $\{i, j, k\}$ is an even permutation of $\{1,2,3\}$; and five $d$ waves with $l=2$,

$$
n_{i}^{\alpha} n_{i}^{\beta}-\frac{1}{3} T^{\alpha \beta} \quad(i=2,3), \quad n_{i}^{\alpha} n_{j}^{\beta}+n_{j}^{\alpha} n_{i}^{\beta} \quad(i \neq j) .
$$

[1] A. Esposito, A. Pilloni, and A. D. Polosa, Phys. Rep. 668, 1 (2017).

[2] R. F. Lebed, R. E. Mitchell, and E. S. Swanson, Prog. Part. Nucl. Phys. 93, 143 (2017).

[3] H.-X. Chen, W. Chen, X. Liu, and S.-L. Zhu, Phys. Rep. 639, 1 (2016).

[4] A. Ali, J. S. Lange, and S. Stone, Prog. Part. Nucl. Phys. 97, 123 (2017).

[5] F.-K. Guo, C. Hanhart, U.-G. Meißner, Q. Wang, Q. Zhao, and B.-S. Zou, Rev. Mod. Phys. 90, 015004 (2018).

[6] S. L. Olsen, T. Skwarnicki, and D. Zieminska, Rev. Mod. Phys. 90, 015003 (2018).

[7] S. K. Choi et al. (Belle Collaboration), Phys. Rev. Lett. 91, 262001 (2003).

[8] D. Acosta et al. (CDF Collaboration), Phys. Rev. Lett. 93, 072001 (2004).

[9] V. M. Abazov et al. (D0 Collaboration), Phys. Rev. Lett. 93, 162002 (2004).

[10] B. Aubert et al. (BABAR Collaboration), Phys. Rev. D 71, 071103 (2005).

[11] R. Aaij et al. (LHCb Collaboration), Eur. Phys. J. C 72, 1972 (2012).

[12] R. Aaij et al. (LHCb Collaboration), Phys. Rev. Lett. 110, 222001 (2013).

[13] G. Barucca et al. (PANDA Collaboration), Eur. Phys. J. A 55, 42 (2019).

[14] M. B. Voloshin, Prog. Part. Nucl. Phys. 61, 455 (2008).

[15] S. Prelovsek, T. Draper, C. B. Lang, M. Limmer, K.-F. Liu, N. Mathur, and D. Mohler, Phys. Rev. D 82, 094507 (2010).

[16] J. Berlin, A. Abdel-Rehim, C. Alexandrou, M. Dalla Brida, M. Gravina, and M. Wagner, Proc. Sci., LATTICE2014 (2014) 104 [arXiv:1410.8757].
Similarly, the 27 combinations $n_{i}^{\alpha} n_{j}^{\beta} n_{k}^{\gamma}$ produce one $s$ wave $\epsilon \varepsilon^{\alpha \beta \gamma \delta} n_{4}^{\delta}$ as well as further $p, d$ and $f$ waves.

Putting these eigenfunctions back into the tensor basis yields the partial-wave decomposition of the amplitude; for example for the $s$ waves,

$$
\begin{aligned}
n_{i}^{\mu} \not h_{j} \otimes \mathbb{1} & \rightarrow T^{\mu \alpha} \gamma^{\alpha} \otimes \mathbb{1}=\gamma_{\perp}^{\mu} \otimes \mathbb{1}, \\
n_{i}^{\mu} \mathbb{1} \otimes \not h_{k} & \rightarrow T^{\mu \beta} \mathbb{1} \otimes \gamma^{\beta}=\mathbb{1} \otimes \gamma_{\perp}^{\mu}, \\
n_{i}^{\mu} h_{j} \otimes \not h_{k} & \rightarrow \epsilon \varepsilon^{\mu \alpha \beta \gamma} n_{4}^{\gamma} \gamma^{\alpha} \otimes \gamma^{\beta} .
\end{aligned}
$$

Combined with the 16 tensors $\Lambda_{\lambda} \Omega_{\omega} \gamma_{5} C \otimes C^{T} \gamma_{5} \Omega_{\omega^{\prime}} \Lambda_{\lambda^{\prime}}$ from Eq. (A6), this yields $48 s$-wave basis elements which are Fierz complete. The tensors in Eq. (15) project onto a subset of these. Since they carry $l=0$, their dressing functions capture the dominant momentum dependence of the axialvector tetraquark amplitude.
[17] S.-h. Lee, C. DeTar, H. Na, and D. Mohler (Fermilab Lattice and MILC Collaborations), arXiv:1411.1389.

[18] S. Prelovsek, C. B. Lang, L. Leskovec, and D. Mohler, Phys. Rev. D 91, 014504 (2015).

[19] M. Padmanath, C. B. Lang, and S. Prelovsek, Phys. Rev. D 92, 034501 (2015).

[20] A. Francis, R. J. Hudspith, R. Lewis, and K. Maltman, Phys. Rev. Lett. 118, 142001 (2017).

[21] P. Bicudo, M. Cardoso, A. Peters, M. Pflaumer, and M. Wagner, Phys. Rev. D 96, 054510 (2017).

[22] A. Francis, R. J. Hudspith, R. Lewis, and K. Maltman, Phys. Rev. D 99, 054505 (2019).

[23] L. Leskovec, S. Meinel, M. Pflaumer, and M. Wagner, arXiv:1904.04197.

[24] S. Prelovsek and L. Leskovec, Phys. Rev. Lett. 111, 192001 (2013).

[25] G. Eichmann, C. S. Fischer, and W. Heupel, Phys. Lett. B 753, 282 (2016).

[26] G. Eichmann, P. Duarte, M. T. Peña, and A. Stadler, arXiv:1907.05402.

[27] O. A. Yakubovsky, Sov. J. Nucl. Phys. 5, 937 (1967).

[28] K. Huang and H. A. Weldon, Phys. Rev. D 11, 257 (1975).

[29] A. M. Khvedelidze and A. N. Kvinikhidze, Theor. Math. Phys. 90, 62 (1992).

[30] W. Heupel, G. Eichmann, and C. S. Fischer, Phys. Lett. B 718, 545 (2012).

[31] G. Eichmann, H. Sanchis-Alepuz, R. Williams, R. Alkofer, and C. S. Fischer, Prog. Part. Nucl. Phys. 91, 1 (2016).

[32] H. Sanchis-Alepuz and R. Williams, Comput. Phys. Commun. 232, 1 (2018).

[33] G. Eichmann, C. S. Fischer, E. Weil, and R. Williams, arXiv:1903.10844.

[34] P. Maris and P. C. Tandy, Phys. Rev. C 60, 055214 (1999). 
[35] G. Eichmann, C. S. Fischer, and W. Heupel, Phys. Rev. D 92, 056006 (2015).

[36] R. Williams, arXiv:1804.11161.

[37] A. S. Miramontes and H. Sanchis-Alepuz, Acta Phys. Pol. B Proc. Suppl. 11, 537 (2018).

[38] M. Tanabashi et al. (Particle Data Group), Phys. Rev. D 98, 030001 (2018).
[39] F. E. Serna, B. El-Bennich, and G. Krein, Phys. Rev. D 96, 014013 (2017).

[40] T. Hilger, M. Gomez-Rocha, A. Krassnigg, and W. Lucha, Eur. Phys. J. A 53, 213 (2017).

[41] C. Hanhart, J. R. Pelaez, and G. Rios, Phys. Lett. B 739, 375 (2014).

[42] G. Eichmann, Phys. Rev. D 84, 014014 (2011). 